\title{
Molecular Links between Alcohol and Tobacco Induced DNA Damage, Gene Polymorphisms and Patho-physiological Consequences: A Systematic Review of Hepatic Carcinogenesis
}

\author{
Abdul Anvesh Mansoori, Subodh Kumar Jain*
}

\begin{abstract}
Chronic alcohol and tobacco abuse plays a crucial role in the development of different liver associated disorders. Intake promotes the generation of reactive oxygen species within hepatic cells exposing their DNA to continuous oxidative stress which finally leads to DNA damage. However in response to such damage an entangled protective repair machinery comprising different repair proteins like ATM,ATR, H2AX, MRN complex becomes activated. Under abnormal conditions the excessive reactive oxygen species generation results in genetic predisposition of various genes (as ADH, ALDH, CYP2E1, GSTT1, GSTP1 and GSTM1) involved in xenobiotic metabolic pathways, associated with susceptibility to different liver related diseases such as fibrosis, cirrhosis and hepatocellular carcinoma. There is increasing evidence that the inflammatory process is inherently associated with many different cancer types, including hepatocellular carcinomas. The generated reactive oxygen species can also activate or repress epigenetic elements such as chromatin remodeling, non-coding RNAs (micro-RNAs), DNA (de) methylation and histone modification that affect gene expression, hence leading to various disorders. The present review provides comprehensive knowledge of different molecular mechanisms involved in gene polymorphism and their possible association with alcohol and tobacco consumption. The article also showcases the necessity of identifying novel diagnostic biomarkers for early cancer risk assessment among alcohol and tobacco users.
\end{abstract}

Keywords: Chronic alcohol consumption - reactive oxygen species - DNA damage - inflammatory cytokines

Asian Pac J Cancer Prev, 16 (12), 4803-4812

\section{Introduction}

The graph of Gastrointestinal (GI) tract malignancies increasing day by day in the world and are increasingly reported in the Indian subcontinent (Ferlay et al., 2010). In the last 5 years, about 1 million new cases of cancers were registered in India reported by International Agency for Research on Cancer (Park et al., 2008; Jemal et al., 2011). The most widespread form of GI cancer in India is of liver origin, which accounts for about $3 \%$ of the total cancer deaths. Out of the total diagnosed cases, 20144 incidences were of liver cancers only. (Bhargava et al., 2012; Bray et al., 2012). Liver carcinoma, an extremely severe condition with underprivileged diagnosis and poor survival is most widespread in central Indian population (Ferlay et al., 2010; Bray et al., 2012). The epidemiological statistics signifies the global rise in the Liver cancer cases particularly in a developing country like India.

Alcohol, mainly ethanol, is by far the most abused drug for centuries globally. The types of alcohol consumed in alcoholic beverages include wine, spirits, liquors, beers and as traditional brew especially in developing countries (Al-Azri et al., 2014). According to the WHO 2014 report, around $30 \%$ of the total population of India consumed alcohol in the year 2010. About $93 \%$ of alcohol was consumed in the form of spirits, followed by beer with $7 \%$ and less than $1 \%$ of the population consumed wine (WHO, 2014). In addition to alcohol, tobacco is also included in carcinogen that causes dysfunction of various genes as well as enzymes involved in detoxification of alcohol and nicotine, consequences in generating various types of liver related diseases (LRD) such as fibrosis, alcoholic hepatitis, cirrhosis, hepatocellular carcinoma (HCC) (Koh et al., 2011; Su et al., 2013).

Epidemiologic studies of the last decades have unequivocally acknowledged chronic alcohol consumption as an important risk factor for the development of various types of cancers, including cancers of the organs and tissues of the respiratory tract and the upper digestive tract (i.e. upper aerodigestive tract), liver, colon or rectum (i.e. 
colorectum), and breast (de Menezes et al., 2013; Kar, 2014). Heavy drinking (i.e. consumption of more than $80 \mathrm{~g}$ alcohol, or more than five to six drinks per day), especially combined with smoking, enhances the risk of developing these cancers by a factor of 50 or more, depending on the population studied (Tong et al., 2014). But some people develop cancer even at relative moderate daily alcohol consumption. These observations suggest that a genetic predisposition may influence cancer risk. At least part of this genetic predisposition may be related to alcohol metabolism because the rate of alcohol metabolism is genetically determined.

According to meta-analysis of sub-population $85 \%$ $90 \%$ of primary liver cirrhosis causes cancers (El-Serag and Rudolph, 2007) accounting for 3.5\% and 7.5\% of all cancers among women and men, respectively (Poustchi et al., 2010; Tong et al., 2014) and accounts for half a million deaths per year (Kirk et al., 2006). Multiple nonviral factors have been concerned with the development of liver cancer include iron overload syndromes, alcohol use, tobacco, oral contraceptive, aflatoxin, pesticides exposure and betel quid chewing, a prevalent habit in the developing world (Gao et al., 2012; Hamed and Ali, 2013; Arora et al., 2015).

\section{Alcohol metabolism through xenobiotics}

A Cancer Preventive Pathway: The xenobioticmetabolizing machinery contains two main types of enzymes: Phase I - mediating oxidative metabolism, and Phase II - conjugating enzymes. Many compounds are transformed to reactive electrophilic metabolites (i.e. ROS) by the oxidative Phase I enzymes, which are mainly alcohol dehydrogenase (ADH), aldehyde dehydrogenase (ALDH) and cytochrome P-450 enzymes (CYPs). Phase II conjugating enzymes, such as glutathione-S-transferase (GST), act usually as inactivating enzymes that inactivate carcinogens (Zakhari, 2013, Yamada et al., 2014).

Ethanol is metabolized by various enzymes including the ADH enzyme isoforms, ALDH and cytochrome P450 enzyme system. CYP2E1 produces reactive oxygen species (ROS), which increase the risk of tissue damage especially to the liver hepatocytes. ROS includes hydroxyethyl, superoxide anion, hydroxyl radicals and number of free radicals (Zakhari, 2013). CYP2E1 which exacerbates some of the toxic effects of acetaldehyde, leads to a harmful condition called oxidative stress in the cells that is characterized by excess levels of ROS (shown in Figure 1). Glutathione S-transferases (GSTs) are a multi-gene family of phase-II metabolic enzymes. The conjugation of reduced glutathione is catalyzed by GSTs enzymes with a variety of endogenous and exogenous electrophilic compounds, including several potentially toxic carcinogens and chemotherapeutic drugs (Yamada et al., 2014), thereby reducing the reactivity of the compounds by making them water soluble and favouring their elimination from the body.

In the body, particularly in the liver cells xenobiotic pathways engender a number of potentially harmful byproducts that cause deleterious possessions on the body tissues and organs. Acetaldehyde, a reactive metabolite of ethanol, binds to nucleic acids, proteins such as enzymes, microsomal proteins and microtubules (Gupta et al., 2014). The generated ROS can also activate or repress the epigenetic mechanisms such as chromatin remodeling, non-coding RNAs (microRNAs), DNA (de) methylation and histone modification that affect gene expression, hence leading to causes various liver diseases and many other disorders (Day and Sweatt, 2011; Gao et al., 2012). It damages DNA leading to chromosome-breakage disease that causes developmental defects, sterility, bone-marrow failure and a highly elevated risk of cancer and other body defects (Nourazarian et al., 2014; Orsetti et al., 2014).

\section{Molecular Regulation of Tobacco Metabolism \& Cancer Prevention}

Tobacco exposure while smoking includes major classes of carcinogenic compounds such as polycyclic aromatic hydrocarbons (PAHs), aromatic amines, nitrosamines, and heterocyclic amines (HCAs); these carcinogenic compounds can enter the alimentary tract or the circulatory systems (Senthilkumar and Thirumurugan, 2012). These carcinogenic compounds are then catalized by CYPs (CYP1A1, CYP1A2, CYP2E1, CYP2A6), leading to DNA-adduct formation or by GSTs (GSTM1, GSTT1, GSTP1) leading to excretion (Koh et al., 2011; Gao et al., 2014).

Nicotine contents of the tobacco leaves can be absorbed in body tissues including skin, respiratory epithelium, and mucous membrane of the mouth. The carcinogenetic potential of tobacco is well-known. Exposure of Nicotine leads to the activation of nicotinic acetylcholine receptors (nAChRs) and may contribute to cancer succession (shown in Figure 1); coupled with the fact that as tumors development there is an increased expression of $\mathrm{nAChRs}$, further representing the connection of this pathway during carcinogenesis (Russo et al., 2012;

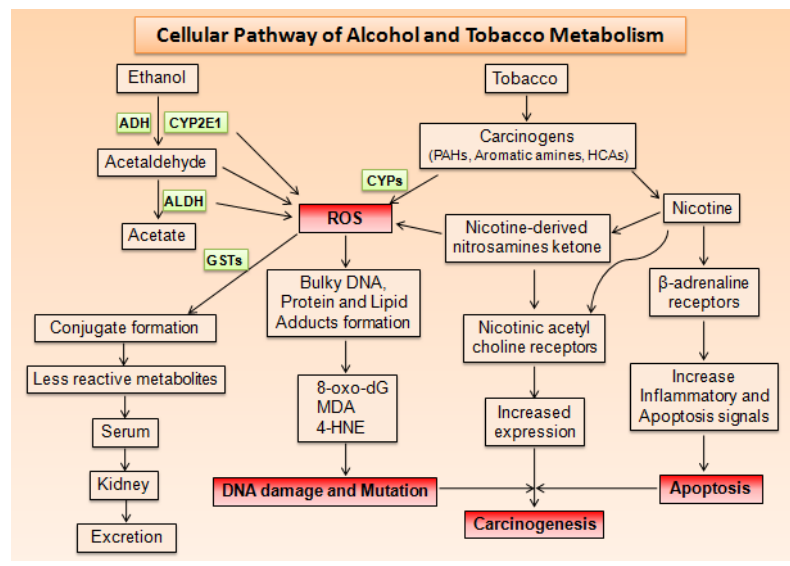

Figure 1. Cellular Pathway of Alcohol and Tobacco Metabolism. Short summary about the link between alcohol and tobacco metabolism by xenobiotic enzymes such as alcohol dehydrogenase (ADH), aldehyde dehydrogenase (ALDH), cytochrome P-450 enzyme (CYP2E1) and glutathione-Stransferase enzymes (GSTs) for the generation of ROS that induced adducts formation(i.e. 8-Oxo-2'-deoxyguanosine (8-oxo-dg), malondialdehyde (MDA), 4-Hydroxynonenal (4$\mathrm{HNE}$ ), etc.) DNA damage, and apoptosis, which finally results in Cancer 
Alcohol and Tobacco Induced DNA Damage, Gene Polymorphisms and Patho-physiology of Hepatic Carcinogenesis

Senthilkumar and Thirumurugan, 2012). Nitrosamines, such as nicotine-derived nitrosamine ketone (NNK), are high affinity ligands for the nAChR signalling and have been shown to enhance intracellular ROS levels in liver cells (Ye et al., 2004).

\section{Identifying diverse form of Tobacco Exposures}

The exposures of Tobacco are of diverse forms. Some people smoked tobacco in the form of cigarette or Bidi, while other chewed in the form of gutkha.

Cigarette Smoke (CS): CS contains p-benzoquinone $(\mathrm{p}-\mathrm{BQ})$ which is derived from $\mathrm{p}$-benzosemiquinone (p-BSQ), produces ROS that is accompanied by inflammation and apoptosis (Panda and Chatterjee, 2007, Banerjee et al., 2008). ROS produced by $\mathrm{p}-\mathrm{BQ}$ is a redox cycling agent that leading to oxidative damage (Margaret et al., 2011). Since CYP2E1 induction is associated with ROS generation and lipid peroxidation, this may be a mechanism whereby tobacco smoke may contribute to HCC. Several studies have suggested that tobacco smoking is a significant risk factor for the development of HCC (Lee et al., 2009; Zygogianni et al., 2011; Purohit et al., 2013). Gajalakshmi and Kanimozhi, (2015) has done a case control study that showed a synergistic interaction between heavy alcohol consumption and tobacco smoking in the progression of HCC.

Tobacco and Gutkha Chewer: About forty percent of the tobacco consumed in India is in the smokeless form such as Pan, Pan masala, Zarda and Gutkha (Gajalakshmi and Kanimozhi, 2015). Almost 70-80 percent of gutkha contains some specific alkaloids named arecoline present in areca nut. According to International Agency for Research on Cancer (IARC 2004), areca nut chewing has been classified to be carcinogenic. Patel et al., (2009) studied harmful effects of tobacco chewing

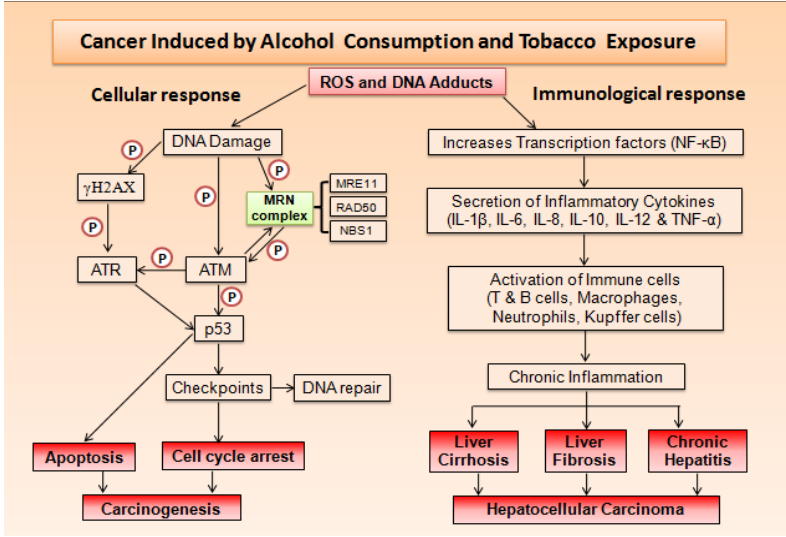

Figure 2. Cancer Induced by Alcohol Consumption and Tobacco Exposure. Mechanism underlying activation of Cellular and Immunological responses which includes activation of DNA damage checkpoints and repair proteins (such as Ataxia-telangiectasia mutated (ATM), Ataxia-telangiectasia Rad 3-related (ATR), MRN complex (Mre11, RAD50 and NBS1 proteins complex), Histone $\mathrm{H} 2 \mathrm{~A}$ isoform $\gamma$ protein $(\gamma \mathrm{H} 2 \mathrm{AX})$, p53 protein), activation of immune cells for the secretion of various class of Interleukins (ILs) \& Tumor necrosis factors (TNF- $\alpha$ ) which induces chronic inflammation in liver that causes cancer in later stages on chromosome integrity in healthy tobacco chewers and found a significant increase in frequency of genetic toxicity in cell (as DNA damage, chromosomal aberration (CA), sister chromatid exchanges, micronucleated cells (SCE) etc. of chewers over controls. A large number of studies related to genotoxic effects of pan masala (Gandhi and Kaur, 2000; Fareed et al., 2011) and gutkha (Jyoti et al., 2011; Chadha and Yadav, 2011) has been reported in literature.

Tobacco Dust Inhale by Bidi Roller: Bidi (the Indian equivalent of a cheap cigarette) rolling is a common cottage industry in India. The bidi rollers are from low socio-economic strata and often work in ill-ventilated confined environments in their dwellings. So they inhale a lot of tobacco dust which leads to many systemic diseases and this is also harmful for the integrity of their chromosomes and DNA (Umadevi et al., 2003, Shukla et al., 2011). Bidi rollers (mostly women) are exposed to tobacco constituents mainly nicotine through the cutaneous route or through inhalation of tobacco dust (Poonam et al., 2010). The amount of tobacco dust may be having cumulative effects on their genetic material with the years of exposure and will cause oxidative damage on lipid, protein, carbohydrate, and nucleic acid molecules (Margaret et al., 2011, Sundaramoorthy et al., 2013). Among the most exploited working groups in the country are Bidi rollers and Tendu pluckers comprising women and children earning bare minimum wages and pathetic to access healthcare facilities (Karabi and Bhavna, 2010). Khanna et al. (2014) investigated that bidi rollers seem to be facing the occupational hazard of genotoxicity due to handling bidi tobacco and inhalation of tobacco dust. They should be advised to work under well-ventilated conditions. In this study the selected bidi rollers and controls were neither smoker nor did they indulge in tobacco chewing.

\section{Cancer Induced by Alcohol Consumption and Tobacco Exposure}

Generated ROS and adducts formation causes structural and functional alternations to DNA that causes cell cycle arrest or apoptosis. This damage severely affects the gene function, such as replication and transcription, and plays a major role in age-related diseases and cancer (Goldar et al., 2015). Continuous oxidative stress induced production of various cytokines, activation of immune cells and finally results in inflammation that become chronic in later stage (shown in Figure 2).

\section{Cellular Responses}

Upshot of Cancer: DNA damage generate a cellular response which is a complex process that includes detection of the DNA damage, activation of signaling pathways including cell cycle checkpoints, and repair of the damage (Niida and Nakanishi, 2006; Seitz and Mueller, 2015). A complex and entangled network of DNA damage response (DDR) mechanisms act as cellular defence against DNA injuries and are able to remove the vast majority of injuries from the genome (Shin et al., 2008; Areeshi, 2013). Each DNA repair mechanism work with 
its own damage specificity. The biological significance of a functional DDR for human health is clearly illustrated by the severe outcomes of inherited defects in DDR factors resulting in various diseases (Shin et al., 2008; Hoeijmakers, 2009).

MRN Complex Association: The MRN complex, whose core comprises of Mre11, RAD50 and NBS1 proteins and is involved in the initial processing of DNA double-strands breaks (DSBS) due to its nuclease activity and DNA binding capability (Uziel et al., 2003). This complex acts as a break sensor and recruits the protein kinase, ataxia telangiectasia mutated (ATM), to DSB sites, facilitating the subsequent processes DNA repair (Lavin, 2007; Lee and Paull, 2007).

The MRN complex adheres to the sites of DSBs instantly following their induction, and this process is independent of ATM (Mirzoeva and Petrini, 2001). It enhances the accumulation of ATM at these sites. NBS1 is important for regulation of the Mre11 complex, influencing DNA binding as well as Mre11 nuclease activity. The activities of the MRN complex are in the very early stage of the DSB response, between damage induction and ATM activation (Stracker et al., 2011).

\section{ATM (Ataxia-Telangiectasia Mutated)}

An important protein play a crucial role in the cellular response to DNA damage is the ataxia telangiectasia mutated (ATM) protein. Conformational changes occur due to DSBs in the ATM protein that stimulates the kinase to phosphorylate Ser1981 by intermolecular autophosphorylation (Shiloh, 2003; Farooqi et al., 2014). After activation, the phosphorylated ATM monomers accumulates at the DNA damage sites and phosphorylates a number of proteins involved in cell cycle checkpoint control, apoptotic responses and DNA repair, including p53, H2AX, Rad17, Nbs1 as well as itself (Shiloh, 2006; Farooqi et al., 2014).

ATM kinase activity is also governed by binding to MRE11 that enhances its ability to phosphorylate itself. A recent study also evidence that autophosphorylation at serine 1981 is essential for monomerization and chromatin association of ATM (Berkovich et al., 2007). Phosphorylation of these and other substrates by ATM initiates cell-cycle arrest at G1/S, intra-S and G2/M checkpoints and also promotes DNA repair (Lavin and Kozlov, 2007). ATM is also known as the caretaker of the genome in humans and act as a central mediator of responses to DNADSBs in cells. Mutations in ATM cause genomic instability syndrome termed ataxia telangiectesia, a rare autosomal recessive disease characterized by immunodeficiency, genome instability and predisposition to cancer in humans (Niida and Nakanishi, 2006; Chaudhary et al., 2013).

\section{ATR (Ataxia-Telangiectasia Rad 3-related)}

Like ATM, ATR kinase activity is responsible for phosphorylation of substrates but its functions may be largely dependent on its subcellular localization. ATR exists in a stable complex with ATR-interacting protein (ATRIP) in human cell, a potential regulatory partner (Unsal-Kacmaz and Sancar, 2004). ATRIP protein that is phosphorylated by ATR, determines ATR expression, and is a critical component of the DNA damage checkpoint pathway. ATR and ATRIP both confine to intra-nuclear foci after DNA damage or inhibition of replication (Wu et al., 2014).

Once ATR is activated then it is translocated to DNA replication foci where activated ATR phosphorylates Chk1, initiating a signal transduction cascade that finally results in cell cycle arrest. Therefore, ATR appears to be a multi-functional kinase that regulates several distinct events from $S$ phase to $M$ phase. Another function of ATR is thought to function in unperturbed DNA replication after activating the DNA damage checkpoint (Chaudhary et al., 2013). ATR is considered as a second checkpointactivating kinase, after ATM, which is activated by double strand breaks in DNA or chromatin disruption (Ohashi et al., 2014).

\section{$\gamma H 2 A X$ (Histone H2A Isoform $\gamma$ Protein)}

Phosphorylated H2AX is also known as $\gamma$-H2AX. Falck et al. (2005) evicted that at the sites of DNA-DSBs, DDR mechanism responsible for the phosphorylation of the histone variant $\mathrm{H} 2 \mathrm{AX}$ by all three phosphoinositide 3-kinases (PIKs)-ATM, ATR and DNA-PK (DNAdependent protein kinase) (Sharma et al., 2015). The phosphorylation of H2AX at C-terminal Serine residues (Ser136 and Ser139) is a relatively early event immediately occurs after DNA damage. $\gamma$-H2AX is also responsible for MRN complex binding and ATM activation (FernandezCapetillo et al., 2004). It also promote efficient repair (Hu et al., 2015) by facilitating the accumulation of components of DNA repair, including NBS1/Mre11/ RAD50 and 53BP1 at damage loci to ensure proper cell cycle arrest or DNA repair.

After DNADSB damage, $\gamma$-H2AX is mainly mediated by ATM, evoking that $\mathrm{H} 2 \mathrm{AX}$ is a downstream mediator of ATM function (Guo et al., 2015). But H2AX is also essential for the recruitment of 53BP1 to sites of DNA DSB damage, and 53BP1 appears to be an upstream activator of ATM (Hu et al., 2015). Therefore, it remains a question whether $\mathrm{H} 2 \mathrm{AX}$ plays any role in activating ATM after DNA DSB damage.

\section{p53 Binding Protein}

The p53 tumor suppressor protein is also known as guardian of the genome, integrates various physiological signals in mammalian cells (Charni et al., 2014). In reverberation to DNA damage, p53 becomes functionally operative and take action either a transient cell cycle arrest, cell death (apoptosis) or permanent cell cycle arrest (cellular senescence). Both apoptosis and cellular senescence are robust tumor suppressor mechanisms that irreversibly forestall damaged cells from undergoing neoplastic transformation (Rodier et al., 2007; Callen et al., 2013). The amount p53 protein and its transcriptional activity is determined by post-translational modification, such as phosphorylation, sumorylation, neddation and acetylation (Charni et al., 2014).

DNA damage causes phosphorylation of p53 at several sites in its transactivation domain, including at Ser15 and Ser20. Activated ATM and ATR phosphorylate p53 
Alcohol and Tobacco Induced DNA Damage, Gene Polymorphisms and Patho-physiology of Hepatic Carcinogenesis

protein at Ser15 residue, resulting in p53 stabilization (Choi et al., 2012; Callen et al., 2013). Various recent studies reported that 53BP1 has been implicated in DNA damage responses and plays a central to both the $\mathrm{S}$ and G2 checkpoints and also responsible for G1 arrest after encounter of DNA damages. The main transcriptional target of p53 is the p21 Cdk inhibitor (p21CKI), which inhibits cyclin E-Cdk2 activity, thereby inhibiting G1/S transition (Lee et al., 2014; Malakar et al., 2014). However, accentuated apoptosis may ultimately lead to liver pathologies, primarily steatosis, which can advance into a more severe disease such as steatohepatitis, fibrosis, and cirrhosis (Charni et al., 2014).

\section{Patho-Physiological Responses}

Causes Cancer progression: Diverse population-based studies suggested that individuals who are prone to chronic inflammatory disorders in liver have an increased risk of liver related diseases development (Gao and Bataller, 2011). In response to a varied range of cellular stresses cytokines are released including infection, inflammation, and carcinogen-induced injury. Host responses that are stimulated by cytokines aimed at controlling cellular stress and minimizing cellular damage (Chung and Lim, 2015). An injury which fails to resolve can elicit excessive immune cell infiltration and lead to persistent cytokine production. Therefore, the host response to stress provokes changes in cytokine expression, which can impact several stages of cancer formation and progression.

\section{Nuclear Factor- $-B(N F-\varkappa B)$}

In the past decade, a number of inflammatory mediators have been shown to contribute to the progression of CLD, many of which are either targets or activators of nuclear factor- $x \mathrm{~B}(\mathrm{NF}-x \mathrm{~B})$. NF-kB is maintained in an inactivated or resting state by the inhibitory kappa $\mathrm{B}(\mathrm{IkB})$ in the cytoplasm. After the phosphorylation of IkB by inhibitor kappa kinase (IkK), NF-kB is activated and free to translocate the nucleus where it can perform its function (Zhang et al., 2015). NF- $x \mathrm{~B}$ is a main transcriptional regulator of the inflammatory response, and plays an important role in the regulation of inflammatory signalling pathways in the liver.

In almost every chronic liver disease NF- $x \mathrm{~B}$ is activated, including alcoholic liver disease, viral hepatitis and biliary liver disease (Mandrekar and Szabo, 2009; Yin et al., 2013). NF- $x$ B regulates multiple important functions in hepatocytes, Kupffer cells and hepatic stellate cells (HSCs). Genetic inactivation of different NF $x \mathrm{~B}$ signalling components results in liver phenotypes that include spontaneous injury, fibrosis and carcinogenesis suggesting that $\mathrm{NF}-\varkappa \mathrm{B}$ makes an essential contribution to liver homeostasis and wound-healing processes (Yao et al., 2014). Activated NF- $x$ B stimulates Toll like receptors (TLRs), as well as inflammatory cytokines such as tumor necrosis factor (TNF) or interleukin (IL)-1 (Yin et al., 2013).

\section{Tumor Necrosis Factor alpha (TNF- $\alpha)$}

TNF- $\alpha$ is pleiotropic growth factor produced by Kupffer cells and other immune cells in response to chronic inflammation and tissue injury. Pro-inflammatory TNF-alpha is connected with an increase in cell cycle progression and oxidative stress through the formation of 8-oxo-deoxyguanosine, a well-known marker of DNA damage associated with chronic hepatitis in human liver (Wang et al., 2012).

Afshar et al. (2015) studied the effect of chronic alcohol consumption in HCV core-expressing transgenic mice and observed that hepatic expression of TGF- $\beta$ and TNF- $\alpha$ has been raised. In another study, higher levels of TNF- $\alpha$ was detected in the tissue surrounding HCC and hepatic metastasis than in the tumor (Park et al., 2010; Chung and Lim, 2015). TNF- $\alpha$ polymorphism produces the TNF- $\alpha$ (-308bp) SNP (single nucleotide polymorphisms) in the promoter region of the gene, which includes TNF- $\alpha 1$ (-308G) and TNF- $\alpha 2$ (-308A) alleles, is linked with cancer susceptibility and induced expression of TNF- $\alpha$ (Talaat et al., 2012; Cen and Wu, 2013).

\section{Pro-inflammatory Cytokines}

Many scientists and researchers all over the world reviewed that cytokines play crucial role in liver carcinoma (Bei et al., 2014; Chung and Lim, 2015). They suggested that cytokines are concerned in liver development and regeneration but their serum level may also contribute to the potential biomarker for pathogenesis of liver-related diseases such as cirrhosis, fibrosis, hepatitis, cancer etc. (Table 1) caused by chronic consumption of alcohol and tobacco abuses. So, one can explore the correlation between expression of cytokines and the different phases of alcoholic liver disease.

\section{Genetic Polymorphism}

Susceptibility to Cancer: Many of the enzymes (such as ADH, ALDH, CYP2E1, GSTM1, GSTT1 and GSTP1) tangled in metabolic xenobiotic pathways have recently been shown to evince genetic polymorphism in the population. ROS and adducts formation is responsible for the predisposition of gene that causes genetic polymorphism (Munaka et al., 2003; Heit et al., 2015).

Table 1. Increased Level of Inflammatory ammatory Cytokines in Serum Associated with Several Chronic Liver Diseases

\begin{tabular}{llll}
\hline Cytokine & \multicolumn{1}{c}{ HCV/HBV } & \multicolumn{1}{c}{ Carcinoma } & \multicolumn{1}{c}{ Other Liver } \\
\hline Interleukin-1 $\beta$ (IL-1 $\beta)$ & Bortolami et al., 2008 & Kanwal et al., 2011 & Kamo et al., 2013 \\
Interleukin 6 (IL-6) & Andrade et al., 2013 & Yin et al., 2013 & Coskun et al., 2004 \\
Interleukin-8 (IL-8) & Langhans et al., 2013 & Lee et al., 2013 & El-Tayeh et al., 2012 \\
Interleukin 10 (IL-10) & da Silva et al., 2015 & Hsia et al., 2007 & Kitaoka et al., 2003 \\
Interleukin 12 (IL-12) & Zhu et al., 2015 & Lo et al., 2010 & Tung et al., 2010 \\
\hline
\end{tabular}


Alcohol Dehydrogenase (ADH) and Aldehyde Dehydrogenase (ALDH)

Lee et al. (2001) determined genotype and allele frequencies of ALDH2, CYP2E1, ADH2 and ADH3 in Korean male patients with alcoholic cirrhosis, without evidence of liver disease, and nondrinkers by using PCR or PCR-directed mutagenesis followed by restriction enzyme digestion and confirmed the observation that the ALDH2 gene protects against the development of alcoholism.

In a population based study done by Zhang et al. (2007) evaluated the effect of polymorphisms in alcohol metabolizing genes, including ADH1B, ADH1C and ALDH2, on levels of alcohol drinking and susceptibility of stomach cancer in Poland. They suggested that the ALDH2 allele may be functionally deficient in eliminating acetaldehyde and discourage alcohol drinking (Eng et al., 2007; Cichoz-Lach et al., 2010; Gubergrits et al., 2014). Furthermore, heavy drinkers of alcohol who were genetically prone to accumulate acetaldehyde may face an increased risk of gastric cancer (Cao et al., 2010; Ferrari et al., 2012).

\section{Cytochromes P450 2E1 (CYP2E1)}

Bennett et al. (1999) investigated that never-smoking women who are exposed to environmental tobacco smoke (ETS) and develop lung cancer are a genetically susceptible population. They analyzed germline polymorphisms in genes that have been associated with cancer susceptibility and whose products activate (CYP2A1) and detoxify (GSTM1 and GSTT1) chemical carcinogens found in tobacco smoke (Yu et al., 2012).

In Brazil, Rossini et al. (2007) analyzed the risk of esophageal squamous cell carcinoma (ESCC) associated with tobacco and alcohol consumption and with polymorphisms of wild type alleles i.e. CYP2A6 (CYP2A6*2), CYP2E1 (CYP2E1*5B, CYP2E1*6), GSTP1 (Ile105Val), GSTM1 and GSTT1 null genotypes in cases and age and gender-matched controls. There was no risk associated with CYP2A6, CYP2E1 and GSTM1 polymorphisms. In conclusion, this study suggests an opposite role of GSTP1 and GSTT1 polymorphisms for the risk for ESCC. Kury et al. (2007) examined the influence on sporadic colorectal cancers risk of environmental factors co-analyzed with combinations of six single nucleotide polymorphisms located in CYP450 genes mainly related to red meat consumption in France. To investigate whether GSTM1, GSTT1, GSTP1, CYP1A1 and CYP2E1 gene polymorphisms represent risk-modifying factors for ethanol related diseases, a study was conducted involving Brazilian alcoholics and controls with similar ethnic backgrounds (Burim et al., 2004). Results were indicating that persons with these genotypes are genetically more prone to the development of alcoholic pancreatitis and alcoholic cirrhosis, respectively (Lakkakula et al., 2013; Malakar et al., 2014; Yu et al., 2015).

\section{Glutathione S-Transferases (GST)}

Among all classes of GSTs, GSTM1, GSTT1 and GSTP1 polymorphisms are extensively studied worldwide. Homozygous deletions of GSTM1 and GSTT1 genes are common and result in a complete loss of enzyme activity. The frequencies of GSTM1 null alleles display race and ethnic variations, being highest in Europeans (42-60\%) $\&$ Asians (41-63\%) compared with that of Africans (1636\%) (Cotton et al., 2000; Gao et al., 2010). However, the frequency of GSTT1 null genotypes is somewhat less in Europeans (13.31\%) compared with that of Africans (14-57\%) and in Asians (35-48\%) (Sobti et al., 2005; Sharma et al., 2012).

Comparison of the frequencies of GST polymorphisms in South Indian population was done by Vettriselvi et al. (2006). He examined that the GSTM1 and GSTT1 null genotype frequencies were found to be $22.4 \%$ and $17.6 \%$ respectively. Allelic variants of GSTM1, GSTT1, and GSTP1 have been associated with increased risk of various cancers like colorectal, lung, breast, prostate and others (Kiyohara et al., 2000; Vijayalakshmi et al., 2005; Malakar et al., 2012; Senthilkumar and Thirumurugan, 2012; Liu et al., 2013; Shen et al., 2014). Susceptibility to oral cancer by genetic polymorphisms among Indians through tobacco exposure as a risk modulator has been studied. The role of polymorphisms at CYP1A1, GSTM1 and GSTT1 to oral squamous cell carcinoma (OSCC) in a case-control study involving patients with precancerous lesions, cancer patients and age and habit-matched controls have been investigated. Finding shows increased susceptibility to buccal mucosa cancer among individuals carrying these genetic markers. These results support the finding that GSTM1 null genotype is a risk factor to OSCC among Indian tobacco habits; GSTT1 null genotype, however, emerged as a protective factor (Anantharaman et al., 2007; Senthilkumar and Thirumurugan, 2012; Dunna et al., 2013;).

\section{Role of Epigenetic Alternations in Cancer Induction}

Epigenetic alteration includes assortment of chemical modification at molecular level that involve alteration of the DNA (e.g. methylation) and the histone proteins (e.g. methylation, acetylation, phosphorylation, ubiquitinylation, ADP-ribosylation, and sumoylation) (Curtis et al., 2013). The epigenetic profile of the organism directly depends on varied aspects including age, environment, and exposure to toxins (e.g., alcohol and tobacco) (Feil and Fraga, 2012). Oxidative metabolites such as acetaldehyde, acetate, acetyl-CoA, and reactive oxygen species (ROS) are generated through alcohol metabolism under the oxidative pathway can induce tissue-specific epigenetic changes (Shukla and Aroor, 2006; Choudhury and Shukla, 2008; Kang et al., 2015). Ethanol metabolism increases ROS level enhances histone H3 acetylation in hepatocytes (Choudhury et al., 2010) and also increases production of acetyl-CoA significantly, which is used in histone acetylation by histone acetyl transferases (HATs) (Yamashita et al., 2001). Continuous exposure of high dose of alcohol to liver cells noticeably amplifies pro-inflammatory cytokine (such as IL-6 and $\mathrm{TNF} \alpha$ ) responses and was also associated with increased acetylation of histone $\mathrm{H} 3$ and $\mathrm{H} 4$ demonstrated by several 
Alcohol and Tobacco Induced DNA Damage, Gene Polymorphisms and Patho-physiology of Hepatic Carcinogenesis

studies (Kendrick et al., 2010). Finally alcohol exposure has tissue specific and immunological responses, such as influencing cell recruitment to infect or inflamed tissue, varying cytokines production level, impairing antigen presentation, hampering with phagocytosis, or inducing apoptosis and finally causes cancer.

\section{Conclusions for preventing cancer}

Prospective studies have indicated a very strong link between the alcohol consumption and tobacco exposure. This link can be assessed by correlating the activation of DNA damage repair proteins, analysing secretion levels of inflammatory cytokines and status of xenobiotic genes polymorphism. Usually people considered alcohol intake as the major risk factor for the development of liver related disorders. But evidence suggested that both alcohol intake and tobacco exposure equally contribute to liver diseases and also enhances the toxic potency of each other. This review focuses on the DNA damage caused by alcohol and tobacco consumption, risk stratification, professional, educational and public awareness connected with genetic and environmental factors. These are the important components of a new strategic approach to the prevention of alcohol related cirrhosis and cancers.

In recent years, due to advancement in molecular epidemiology, an emerging new field that combines extremely sensitive and specific techniques for detecting early damage associated with cancer, it has been possible to identify risks and thwart adverse health consequences related to environmental exposures by exogenous and endogenous compound. Therefore, this review focuses on the possible relationship between inter-individual differences in gene structure and inducibility of enzymes responsible for xenobiotic metabolism. This information has been used for the evaluation of increased susceptibility for cancer. Furthermore, this review will represent a crucial step to understand the susceptibility to develop cirrhosis and cancer in alcoholics and will be of importance in the social awareness to people and prevention of alcohol related diseases and/or the development of better therapies. It will be also be important to identify new diagnostic biomarkers in future that might allow more straightforward treatment and for identifying high risk individuals at an early stage. Hopefully, this review will implement the researchers and clinicians to foster, explore and authenticate the impacts and underlying mechanisms of DNA damage response (DDR) pathways in liver cirrhosis and cancer.

\section{References}

Afshar M, Richards S, Mann D, et al (2015). Acute immunomodulatory effects of binge alcohol ingestion. Alcohol, 49, 57-64

Al-Azri M, Al-Rasbi K, Al-Hinai M, et al (2014). Awareness of risk factors for cancer among Omani adults--a community based study. Asian Pac J Cancer Prev, 15, 5401-6

Anantharaman D, Chaubal PM, Kannan S, et al (2007). Susceptibility to oral cancer by genetic polymorphisms at CYP1A1, GSTM1 and GSTT1 loci among Indians: tobacco exposure as a risk modulator. Carcinogenesis, 28, 1455-62

Andrade BB, Hullsiek KH, Boulware DR, et al (2013)
Biomarkers of inflammation and coagulation are associated with mortality and hepatitis flares in persons coinfected with HIV and hepatitis viruses. J Infect Dis, 207, 1379-88

Areeshi MY (2013). Genetic variation in a DNA double strand break repair gene in saudi population: a comparative study with worldwide ethnic groups. Asian Pac J Cancer Prev, 14, 7091-4

Arora D, Marya CM, Menon I, et al (2015). Cross sectional survey on association between alcohol, betel- nut, cigarette consumption and health promoting behavior of industrial workers in Ghaziabad. Asian Pac J Cancer Prev, 16, 139-44

Banerjee S, Chattopadhyay R, Ghosh A, et al (2008). Cellular and molecular mechanisms of cigarette smoke-induced lung damage and prevention by vitamin C. J Inflamm, 5, 21

Bei CH, Bai H, Yu HP, et al (2014). Combined effects of six cytokine gene polymorphisms and SNP-SNP interactions on hepatocellular carcinoma risk in Southern Guangxi, China. Asian Pac J Cancer Prev, 15, 6961-7

Bennett WP, Alavanja MCR, Blomeke B, et al (1999). Environmental tobacco smoke, genetic susceptibility, and risk of lung cancer in never-smoking women. J Natl Cancer Inst, 91, 2009-14

Berkovich E, Monnat RJ, Kastan MB (2007). Roles of ATM and NBS1 in chromatin structure modulation and DNA doublestrand break repair. Nat Cell Biol, 9, 683-90

Bhargava A, Mishra D, Banerjee S, et al (2012). Engineered dendritic cells for gastrointestinal tumor immunotherapy: opportunities in translational research. J Drug Target, 21, 126-36.

Bortolami M, Kotsafti A, Cardin R, et al (2008). Fas / FasL system, IL-1beta expression and apoptosis in chronic HBV and HCV liver disease. $J$ Viral Hepat, 15, 515-22

Bray F, Ren JS, Masuyer E, et al (2012). Global estimates of cancer prevalence for 27 sites in the adult population in 2008. Int J Cancer, 2012, 1133-45

Burim RV, Canalle R, Martinelli Ade L, et al (2004). Polymorphisms in glutathione S-transferases GSTM1, GSTT1 and GSTP1 and cytochromes P450 CYP2E1 and CYP1A1 and susceptibility to cirrhosis or pancreatitis in alcoholics. Mutagenesis, 19, 291-298

Callen E, Di Virgilio M, Kruhlak MJ, et al (2013). 53BP1 mediates productive and mutagenic DNA repair through distinct phosphoprotein interactions. Cell, 153, 1266-80

Cao HX, Li SP, Wu JZ, et al (2010). Alcohol dehydrogenase-2 and aldehyde dehydrogenase-2 genotypes, alcohol drinking and the risk for stomach cancer in Chinese males. Asian Pac J Cancer Prev, 11, 1073-7

Cen G, Wu W (2013). Association between tumor necrosis factor-alpha $857 \mathrm{C} / \mathrm{T}$ polymorphism and gastric cancer: a meta-analysis. Tumour Biol, 34, 3383-8

Chadda P, Yadav, JS (2011). Studies on the Genotoxicity of Gutkha. Genet, 11, 277-82

Charni M, Rivlin N, Molchadsky A, et al (2014). p53 in liver pathologies-taking the good with the bad. J Mol Med (Berl), 92, 1229-34

Chaudhary P, Sharma R, Sahu M, et al (2013). 4-Hydroxynonenal induces $\mathrm{G} 2 / \mathrm{M}$ phase cell cycle arrest by activation of the ataxia telangiectasia mutated and Rad3-related protein (ATR)/checkpoint kinase 1 (Chk1) signaling pathway. J Biol Chem, 288, 20532-46

Choi HS, Seo HS, Kim JH, et al (2012). Ethanol extract of Paeonia Suffruticosa Andrews (PSE) induced AGS human gastric cancer cell apoptosis via fas-dependent apoptosis and MDM2-p53 pathways. J Biomed Sci, 19, 82

Choudhury M, Park PH, Jackson D, Shukla SD (2010). Evidence for the role of oxidative stress in the acetylation of histone $\mathrm{H} 3$ by ethanol in rat hepatocytes. Alcohol, 44, 531-40 
Choudhury M, Shukla SD (2008). Surrogate alcohols and their metabolites modify histone $\mathrm{H} 3$ acetylation: Involvement of histone acetyl transferase and histone deacetylase. Alcohol Clin Exp Res, 32, 829-39

Chung HW, Lim JB (2015). Role of the tumor microenvironment in the pathogenesis of gastric carcinoma. World $J$ Gastroenterol, 20, 1667-80

Cichoz-Lach H, Celinski K, Wojcierowski J, et al (2010). Genetic polymorphism of alcohol-metabolizing enzyme and alcohol dependence in Polish men. Braz J Med Biol Res, 43, 257-61

Coskun U, Bukan N, Sancak B, et al (2004). Serum hepatocyte growth factor and interleukin-6 levels can distinguish patients with primary or metastatic liver tumors from those with benign liver lesions. Neoplasma, 51, 209-13

Cotton SC, Sharp L, Little J, et al (2000). Glutathione S-transferase polymorphisms and colorectal cancer: a HuGE review. Am J Epidemiol, 151, 7-32

Curtis BJ, Zahs A, Kovacs EJ (2013). Epigenetic targets for reversing immune defects caused by alcohol exposure. Alcohol Res, 35, 97-113

da Silva NM, Germano FN, Vidales-Braz BM, et al (2015). Polymorphisms of IL-10 gene in patients infected with $\mathrm{HCV}$ under antiviral treatment in southern Brazil. Cytokine, 73, 253-257

Day JJ, Sweatt JD (2011). Review of Epigenetic Mechanisms in Cognition. Neuron, 70, 813-29

de Menezes RF, Bergmann A, Thuler LC (2013). Alcohol consumption and risk of cancer: a systematic literature review. Asian Pac J Cancer Prev, 14, 4965-72

Dunna NR, Vure S, Sailaja K, et al (2014). Deletion of GSTM1 and $\mathrm{T} 1$ genes as a risk factor for development of acute leukemia. Asian Pac J Cancer Prev, 14, 2221-4

El-Serag HB, Rudolph KL (2007). Hepatocellular carcinoma: epidemiology and molecular carcinogenesis. Gastroenterol, 132, 2557-76

El-Tayeh SF, Hussein TD, El-Houseini ME, et al (2012). Serological biomarkers of hepatocellular carcinoma in Egyptian patients. Disease Markers, 32, 255-63

Eng MY, Luczak SE, Wall TL (2007). ALDH2, ADH1B and ADH1C Genotypes in Asians: A Literature Review. Alcohol Res Health, 30, 22-26

Falck J, Coates J, Jackson SP (2005). Conserved modes of recruitment of ATM, ATR and NA-PKcs to sites of DNA damage. Nature, 434, 605-11

Farooqi AA, Attar R, Arslan BA, et al (2014). Recently emerging signaling landscape of Ataxia-telangiectasia mutated (ATM) kinase. Asian Pac J Cancer Prev, 15, 6485-8

Feil R, Fraga MF (2012). Epigenetics and the environment: Emerging patterns and implications. Nature Rev Genetics, 13, 97-109

Ferlay J, Shin HR, Bray F, et al (2010). Cancer Incidence and Mortality Worldwide: IARC Cancer Base No. 10 [Internet]. Lyon, France: International Agency for Research on Cancer; 2010

Fernandez-Capetillo O, Lee A, Nussenzweig M, et al (2004). H2AX: the histone guardian of the genome. DNA Repair (Amst), 3, 959-67

Ferrari P, McKay JD, Jenab M, et al (2012). Alcohol dehydrogenase and aldehyde dehydrogenase gene polymorphisms, alcohol intake and the risk of colorectal cancer in the European Prospective Investigation into Cancer and Nutrition study. Eur J Clin Nutr, 66, 1303-8

Freed M, Afzal M, Siddique YH (2011). Micronucleus investigation in buccal mucosa cells among pan masala/ gutkha chewers and its relevance for oral cancer. Bio Med, 3, $8-15$

Gajalakshmi V, Kanimozhi V (2015). Tobacco chewing and adult mortality: a case-control analysis of 22,000 cases and 429,000 controls, never smoking tobacco and never drinking alcohol, in South India. Asian Pac J Cancer Prev, 16, 1201-6

Gandhi G, Kaur R (2000) Cytogenetic studies in exfoliated cells of high cancer risk groups, pan masala chewers. Human Ecololgy Special Issue, 9, 221-8

Gao B, Bataller R (2011). Alcoholic liver disease: pathogenesis and new therapeutic targets. Gastroenterol, 141, 1572-85

Gao J, Xie L, Yang WS, et al (2012). Risk factors of hepatocellular carcinoma--current status and perspectives. Asian Pac J Cancer Prev, 13, 743-52

Gao M, Li Y, Xue X, et al (2014). Impact of AhR, CYP1A1 and GSTM1 genetic polymorphisms on TP53 R273G mutations in individuals exposed to polycyclic aromatic hydrocarbons. Asian Pac J Cancer Prev, 15, 2699-705

Gao Y, Cao Y, Tan A, et al (2010). Glutathione S-transferase M1 polymorphism and sporadic colorectal cancer risk: an updating Meta-analysis and HuGe Review of 36 case-control studies. Ann Epidemiol, 20, 108-121

Goldar S, Khaniani MS, Derakhshan SM, Baradaran B (2015). Molecular mechanisms of apoptosis and roles in cancer development and treatment. Asian Pac J Cancer Prev, 16, 2129-44

Gubergrits NB, Kishenya MS, Golubova OA (2014). Polymorphism of ethanol metabolism genes in alcoholic chronic pancreatitis. Ter Arkh, 86, 49-55

GuoZ, Pei S, Si T, et al (2015). Expression of the $\gamma$-phosphorylated histone $\mathrm{H} 2 \mathrm{AX}$ in gastric carcinoma and gastric precancerous lesions. Oncol Lett, 9, 1790-94

Gupta RK, Patel AK, Shah N, et al (2014). Oxidative stress and antioxidants in disease and cancer: a review. Asian Pac J Cancer Prev, 15, 4405-9

Hamed MA, Ali SA (2013). Non-viral factors contributing to hepatocellular carcinoma. World J Hepatol, 5, 311-22

Heit C, Dong H, Chen Y, et al (2015). Transgenic mouse models for alcohol metabolism, toxicity, and cancer. Adv Exp Med Biol, 815, 375-87

Hoeijmakers JH (2009). DNA damage, aging, and cancer. $N$ Engl J Med, 361, 1475-85

Hsia CY, Huo TI, Chiang SY, et al (2007). Evaluation of interleukin-6, interleukin-10 and human hepatocyte growth factor as tumor markers for hepatocellular carcinoma. Eur J Surg Oncol, 33, 208-12

Hu JL, Hu SS, Hou XX, et al (2015). Abnormal expression of DNA double-strand breaks related genes, ATM and gammaH2AX, in thyroid carcinoma. Int $J$ Endocrinol, 2015, 136810 .

IARC (2004) Monographs on the evaluation of the carcinogenic risks to humans, tobacco habits other than smoking; betel quid and areca nut chewing. Lyon: International Agency for Research on Cancer, 85

Jemal A, Bray F, Center MM, et al (2011). Global cancer statistics. CA Cancer J Clin, 61, 69-90

Jyoti S, Afzal M, Siddique YH (2011). Genotoxic effects of pan masala and gutkha: A review. World J Zool, 6, 301-6

Kamo N, Ke B, Ghaffari AA, et al (2013). ASC/caspase-1/IL$1 \beta$ signaling triggers inflammatory responses by promoting HMGB1 induction in liver ischemia/reperfusion injury. Hepatol, 58, 351-62

Kang JY, Song SH, Yun J, et al (2015). Identification of longrange epigenetic silencing on chromosome $15 \mathrm{q} 25$ and its clinical implication in gastric cancer. Am J Pathol, 185, 666-78

Kanwal F, Hoang T, Kramer JR, et al (2011) Increasing prevalence of $\mathrm{HCC}$ and cirrhosis in patients with chronic hepatitis C virus infection. Gastroenterol, 140, 1182-88

Kar P (2014) Risk factors for hepatocellular carcinoma in India. 
Alcohol and Tobacco Induced DNA Damage, Gene Polymorphisms and Patho-physiology of Hepatic Carcinogenesis

J Clin Exp Hepatol, 4, 34-42

Karabi MM, Bhavna MB (2010) Tobacco and poverty research and capacity building: narrative report. organization: voluntary health association of India, New Delhi, India

Kendrick SF, O’Boyle G, Mann J, et al (2010). Acetate, the key modulator of inflammatory responses in acute alcoholic hepatitis. Hepatol, 51, 1988-97

Khanna A, Gautam DS, Gokhale M, Jain, S.K. (2014). Tobacco dust induced genotoxicity as an occupational hazard in workers of bidi making cottage industry of central India Toxicology International, 21, 18-23

Kirk GD, Bah E, Montesano R (2006). Molecular epidemiology of human liver cancer: insights into etiology, pathogenesis and prevention from The Gambia, West Africa. Carcinogenesis, 27, 2070-82

Kitaoka S, Shiota G, Kawasaki H (2003). Serum levels of interleukin-10, interleukin-12 and soluble interleukin-2 receptor in chronic liver disease type C. Hepatogastroenterol, 50, 1569-74

Kiyohara C, Yamamura K, Nakanishi Y (2000). Polymorphism in GSTM1, GSTT1 and GSTP1 and susceptibility to lung cancer in a Japanese population. Asian Pac J Cancer Prev, 1, 293-8

Koh WP, Robien K, Wang R, et al (2011). Smoking as an independent risk factor for hepatocellular carcinoma: the Singapore Chinese health study. Br J Cancer, 105, 1430-5

Küry S, Buecher B, Robiou-du-Pont S, et al (2007). Combinations of cytochrome $\mathrm{P} 450$ gene polymorphisms enhancing the risk for sporadic colorectal cancer related to red meat consumption. Cancer Epidemiol Biomarkers Prev, 16, 1460-67

Lakkakula S, Maram R, Munirajan AK, et al (2013). Functional PstI/RsaI polymorphisms in the CYP2E1 gene among south Indian populations. Asian Pac J Cancer Prev, 14, 179-82

Langhans B, Krämer B, Louis M, et al (2013). Intrahepatic IL-8 producing Foxp $3^{+} \mathrm{CD} 4{ }^{+}$regulatory $\mathrm{T}$ cells and fibrogenesis in chronic hepatitis C. J Hepatol, 59, 229-35

Lavin MF (2007). ATM and the Mre11 complex combine to recognize and signal DNA double-strand breaks. Oncogene, $26,7749-58$

Lavin MF, Kozlov S (2007). ATM activation and DNA damage response. Cell Cycle, 6, 931-42

Lee HC., Lee HS, Jung SH, et al (2001). Association between polymorphisms of ethanol metabolizing enzymes and susceptibility to alcoholic cirrhosis in a Korean male population. J Korean Med Sci, 16, 745-50

Lee HE, Han N, Kim MA, et al (2014). DNA damage responserelated proteins in gastric cancer: ATM, Chk2 and p53 expression and their prognostic value. Pathobiol, 81, 25-35

Lee JH, Paull TT (2007). Activation and regulation of ATM kinase activity in response to DNA double-strand breaks. Oncogene, 26, 7741-48

Lee KH, Koh SA, Kim JR (2013). Hepatocyte growth factormediated gastrin-releasing peptide induces IL- 8 expression through Ets-1 in gastric cancer cells. Oncol Res, 20, 393-402

Lee YC, Cohet C, Yang YC, et al (2009). Meta-analysis of epidemiologic studies on cigarette smoking and liver cancer. Int J Epidemiol, 38, 1497-511

Liu K, Zhang L, Lin X, et al (2013). Association of GST genetic polymorphisms with the susceptibility to hepatocellular carcinoma (HCC) in Chinese population evaluated by an updated systematic meta-analysis. PLoS One, 8, e57043

Lo CH, Chang CM, Tang SW, et al (2010). Differential antitumor effect of interleukin-12 family cytokines on orthotopic hepatocellular carcinoma. J Gene Med, 12, 423-34

Malakar M, Devi KR, Phukan RK, et al (2012). Genetic polymorphism of glutathione S-transferases M1 and T1, tobacco habits and risk of stomach cancer in Mizoram, India. Asian Pac J Cancer Prev, 13, 4725-32

Malakar M, Devi KR, Phukan RK, et al (2014). CYP2E1 genetic polymorphism with dietary, tobacco, alcohol habits, $\mathrm{H}$. pylori infection status and susceptibility to stomach cancer in Mizoram, India. Asian Pac J Cancer Prev, 15, 8815-22

Malakar M, Devi KR, Phukan RK, et al (2014). p53 codon 72 polymorphism interactions with dietary and tobacco related habits and risk of stomach cancer in Mizoram, India. Asian Pac J Cancer Prev, 15, 717-23

Mandrekar P, Szabo G (2009). Signalling pathways in alcoholinduced liver inflammation. J Hepatol, 50, 1258-66

Margaret AL, Syahruddin E, Wanandi SI (2011). Low activity of manganese superoxide dismutase (MnSOD) in blood of lung cancer patients with smoking history: relationship to oxidative stress. Asian Pac J Cancer Prev, 12, 3049-53

Mirzoeva OK, Petrini JH (2001). DNA damage-dependent nuclear dynamics of the Mre11 complex. Mol Cell Biol, 21, 281-8

Munaka M, Kohshi K, Kawamoto T, et al (2003). Genetic polymorphisms of tobacco and alcohol-related metabolizing enzymes and the risk of hepatocellular carcinoma. J Cancer Res Clin Oncol, 129, 355-60

Niida H, Nakanishi M (2006). DNA damage checkpoints in mammals. Mutagenesis, 21, 3-9

Nourazarian AR, Kangari P, Salmaninejad A (2014). Roles of oxidative stress in the development and progression of breast cancer. Asian Pac J Cancer Prev, 15, 4745-51

Ohashi E, Takeishi Y, Ueda S, Tsurimoto T (2014). Interaction between Rad9-Hus1-Rad1 and TopBP1 activates ATRATRIP and promotes TopBP1 recruitment to sites of UVdamage. DNA Repair (Amst), 21, 1-11

Orsetti B, Selves J, Bascoul-Mollevi C, et al (2014). Impact of chromosomal instability on colorectal cancer progression and outcome. BMC Cancer, 14, 121

Panda K, Chatterjee IB (2007). Failure of alpha-tocopherol to prevent cigarette smoke-induce protein oxidation: comparison with other antioxidant vitamins. victor r. editor, encyclopedia of vitamin E. Oxford University Press (CABI), Oxfordshire, UK. Preedy, King's College, London, UK. Chapter, 63, 883-93

Park EJ, Lee JH, Yu GY, et al (2010). Dietary and genetic obesity promote liver inflammation and tumorigenesis by enhancing IL-6 and TNF expression. Cell, 140, 197-208

Park S, Bae J, Nam BH, Yoo KY (2008). Aetiology of cancer in Asia. Asian Pac J Cancer Prev, 9, 371-80

Patel BP, Trivedi PJ, Brahmbhatt MM, et al (2009). Micronuclei and chromosomal aberrations in healthy tobacco chewers and controls: A study from Gujrat, India. Arch of Oncol, 17, 1-2

Poonam P, Khanna A, Jain SK (2010). Evaluation of genotoxicity in Bidi Rollers occupationally exposed to tobacco dust. National J Life Sci, 7, 89-93

Poustchi H, Sepanlou SG, Esmaili S, Mehrabi N, Ansarymoghadam A (2010). Hepatocellular carcinoma in the world and the Middle East. Middle East J Diges Dis, 2, 31-41

Purohit V, Rapaka R, Kwon OS, et al (2013). Roles of alcohol and tobacco exposure in the development of hepatocellular carcinoma. Life Sci, 92, 3-9

Rodier F, Campisi J, Bhaumik D (2007). Two faces of p53: aging and tumor suppression. Nucleic Acids Res, 35, 7475-84

Rossini A, Rapozo DC, Soares Lima SC, et al (2007). Polymorphisms of GSTP1 and GSTT1, but not of CYP2A6, CYP2E1 or GSTM1, modify the risk for esophageal cancer in a western population. Carcinogenesis, 28, 2537-42

Russo P, Cardinale A, Margaritora S, Cesario A (2012). Nicotinic receptor and tobacco-related cancer. Life Sci, 91, 1087-92

Seitz HK, Mueller S (2015). Alcohol and cancer: an overview 
with special emphasis on the role of acetaldehyde and cytochrome P450 2E1. Adv Exp Med Biol, 815, 59-70

Senthilkumar KP, Thirumurugan R (2012). Impact of tobacco on glutathione $\mathrm{S}$ transferase gene loci of Indian ethnics. Asian Pac J Cancer Prev, 13, 5037-42

Senthilkumar KP, Thirumurugan R (2012). Impact of tobacco on glutathione $\mathrm{S}$ transferase gene loci of Indian ethnics. Asian Pac J Cancer Prev, 13, 5037-42

Sharma A, Pandey A, Sardana S, Sehgal S, Sharma JK (2012). Genetic polymorphisms of GSTM1 and GSTT1 genes in Delhi and comparison with other Indian and Global populations. Asian Pac J Cancer Prev, 13, 5647-52

Sharma AK, Bhattacharya S, Khan SA, et al (2015). Dynamic alteration in $\mathrm{H} 3$ serine 10 phosphorylation is G1-phase specific during ionization radiation induced DNA damage response in human cells. Mutat Res, 773, 83-91

Shen YH, Chen S, Peng YF, et al (2014). Quantitative assessment of the effect of glutathione S-transferase genes GSTM1 and GSTT1 on hepatocellular carcinoma risk. Tumour Biol, 35, 4007-15

Shiloh Y (2003). ATM and related protein kinases: safeguarding genome integrity. Nat Rev Cancer, 3, 155-68

Shiloh Y (2006). The ATM-mediated DNA-damage response: taking shape. Trends Biochem Sci, 31, 402-10

Shin A, Lee KM, Ahn B, et al (2008). Genotype-phenotype relationship between DNA repair gene genetic polymorphisms and DNA repair capacity. Asian Pac J Cancer Prev, 9, 501-5

Shukla P, Khanna A, Jain SK (2011). Working condition: A key factor in increasing occupational hazard among bidi rollers: A population health research with respect to DNA damage. Indian J Occup Environ Med, 15, 139-41

Shukla SD, Aroor AR (2006). Epigenetic effects of ethanol on liver and gastrointestinal injury. World JGastroenterol, 12, 5265-71

Sobti RC, Al-Badran AI, Sharma S, et al (2005). Genetic polymorphisms of CYP2D6, GSTM1 and GSTT1 genes and bladder cancer risk in north India. Cancer Genet Cytogenet, 156, 68-73

Stracker TH, Petrini JH (2011). The MRE11 complex: starting from the ends. Nat Rev Mol Cell Biol, 12, 90-103

Su CH, Lin Y, Cai L (2013). Genetic factors, viral infection, other factors and liver cancer: an update on current progress. Asian Pac J Cancer Prev, 14, 4953-60

Sundaramoorthy R, Srinivasan V, Gujar J, et al (2013). Clinical, cytogenetic and CYP1A1 exon-1 gene mutation analysis of Beedi workers in Vellore region, Tamil Nadu. Asian Pac J Cancer Prev, 14, 7555-60

Talaat RM, Esmail AA, Elwakil R, Gurgis AA, Nasr MI (2012). Tumor necrosis factor-alpha -308G/A polymorphism and risk of hepatocellular carcinoma in hepatitis $\mathrm{C}$ virus-infected patients. Chin J Cancer, 31, 29-35

Tong GX, Liang H, Chai J, et al (2014). Association of risk of gastric cancer and consumption of tobacco, alcohol and tea in the Chinese population. Asian Pac J Cancer Prev, 15, 8765-74

Tung KH, Huang YS, Yang KC, et al (2010). Serum Interleukin-12 Levels in Alcoholic Liver Disease. J Chin Med Assoc, 73, 67-71

Umadevi B, Swama M, Padmavathi P, Jyothi A, Reddy PP (2003). Cytogenetic effects in workers occupationally exposed to tobacco dust. Mutat Res, 535, 147-54

Unsal-Kacmaz K, Sancar A (2004). Quaternary structure of ATR and effects of ATRIP and replication protein A on its DNA binding and kinase activities. Mol Cell Biol, 24, 1292-1300

Uziel T, Lerenthal Y, Moyal L, (2003). Requirement of the MRN complex for ATM activation by DNA damage. EMBO J, 22, 5612-21

Vettriselvi V, Vijayalakshmi K, Paul SD, Venkatachalam P
(2006). Genetic variation of GSTM1, GSTT1 and GSTP1 genes in a South Indian population. Asian Pac J Cancer Prev, 7, 325-28

Vijayalakshmi K, Vettriselvi V, Krishnan M, et al (2005). Polymorphisms at GSTM1 and GSTP1 gene loci and risk of prostate cancer in a South Indian population. Asian Pac J Cancer Prev, 6, 309-14

Wang HJ, Gao B, Zakhari S, et al (2012). Inflammation in alcoholic liver disease. Annu Rev Nutr, 32, 343-68

WHO (2014). Global status report on alcohol and health 2014. Publication Data. 2014

Wu CS, Ouyang J, Mori E, et al (2014). SUMOylation of ATRIP potentiates DNA damage signaling by boosting multiple protein interactions in the ATR pathway. Genes Dev, 28, 1472-84

Yamada I, Matsuyama M, Ozaka M, et al (2014). Lack of associations between genetic polymorphisms in GSTM1, GSTT1 and GSTP1 and pancreatic cancer risk: a multiinstitutional case-control study in Japan. Asian Pac J Cancer Prev, 15, 391-5

Yamashita H, Kaneyuki T, Tagawa K (2001). Production of acetate in the liver and its utilization in peripheral tissues. Biochimica et Biophysica Acta, 1532, 79-87

Yao J, Qian CJ, Ye B, et al (2014). Signal transducer and activator of transcription 3 signaling upregulates fascin via nuclear factor- $x \mathrm{~B}$ in gastric cancer: Implications in cell invasion and migration. Oncol Lett, 7, 902-8

Ye YN, Liu ES, Shin VY, et al (2004). The modulating role of nuclear factor-kappa B in the action of alpha7nicotinic acetylcholine receptor and cross-talk between 5lipoxygenase and yclooxygenase- 2 in colon cancer growth induced by 4-(N-methyl-N-nitrosamino)-1- (3-pyridyl)-1butanone. J Pharmacol Exp Ther, 311, 123-30

Yin Y, Si X, Gao Y, et al (2013). The nuclear factor- $x$ B correlates with increased expression of interleukin- 6 and promotes progression of gastric carcinoma. Oncol Rep, 29, 34-8

Yu BW, Zhang LQ, Teng XL, et al (2015). Association between the CYP1A1 polymorphisms and hepatocellular carcinoma: a meta-analysis. Genet Mol Res, 14, 1076-84

Yu L, Sun L, Jiang YF, et al (2012). Interactions between CYP1A1 polymorphisms and cigarette smoking are associated with the risk of hepatocellular carcinoma: evidence from epidemiological studies. Mol Biol Rep, 39, 6641-6

Zakhari S (2013). Bermuda Triangle for the liver: Alcohol, obesity, and viral hepatitis. J Gastroenterol Hepatol, 28, $18-25$

Zhang FF, Hou L, Terry et al (2007). Genetic polymorphisms in alcohol metabolism, alcohol intake and the risk of stomach cancer in Warsaw, Poland. Int J Cancer, 121, 2060-64

Zhang L, Ding Y, Yuan Z, et al (2015). MicroRNA-500 sustains nuclear factor- $x \mathrm{~B}$ activation and induces gastric cancer cell proliferation and resistance to apoptosis. Oncotarget, 6, 2483-95

Zhu B, Wang C, Zhang X, et al (2015). Relationships between interleukin-12B and interleukin-10 gene polymorphisms and hepatitis $\mathrm{C}$ in Chinese Han hemodialysis patients. Ren Fail, 37, 505-10.

Zygogianni A, Kyrgias G, Mystakidou K, et al (2011). Potential role of the alcohol and smoking in the squamous cell carcinoma of the head and neck: review of the current literature and new perspectives. Asian Pac J Cancer Prev, 12, 339-44 